\title{
Intravenously Administered 2'-Deoxycytidine Suppresses Mouse Myeloma Tumor Growth
}

\author{
Ayano Iwazaki, Kimie Imai, Kunio Nakanishi, and Masanori Yoshioka \\ Faculty of Pharmaceutical Sciences, Setsunan University; 45-1 Nagaotoge-cho, Hirakata, Osaka 573-0101, Japan. \\ Received August 18, 2011; accepted November 16, 2011; published online November 18, 2011
}

We examined the in vivo effects of intravenously administered 2'-deoxycytidine (dCyd) on tumor growth and survival time in mice bearing SP2/0-Ag14 (SP2/0) myeloma tumors. Administration of dCyd tended to decrease the tumor volume and significantly decreased the tumor weight. A single intravenous administration of dCyd significantly increased survival time of the tumor-bearing mice. The effect of dCyd on tumor growth was maintained for at least 1 week after the final administration. The net amount of dCyd in the kidney, liver, and spleen of the tumor-bearing mice increased 2.5 to 5.3 fold compared with the amount in nontumor-bearing mice. Our results suggest that the increase in dCyd in the mice inoculated SP2/0 myeloma cells plays an important role for the growth suppression of the tumor.

Key words 2'-deoxycytidine; anti-tumor; survival

The nucleoside 2'-deoxycytidine (dCyd) is metabolized as a material for DNA and RNA synthesis in the body. Some anti-tumor agents have been developed based on the structure of $\mathrm{dCyd}$ because it is essential for the synthesis of nucleic acids. Agents such as $1-\beta$-D-arabinofuranosylcytosine (ara-C), ${ }^{1,2}$ $2^{\prime}, 2^{\prime}$-difluoro-2'-deoxycytidine (gemcitabine), ${ }^{2,3)}$ and 5-aza-2'deoxycytidine (decitabine ${ }^{4)}$ inhibit nucleic acid metabolism. In a previous study, we reported that intraperitoneal administration of dCyd tended to decrease tumor growth in mice subcutaneously transplanted with SP2/0-Ag14 mouse myeloma cells $(\mathrm{SP} 2 / 0)$ to generate solid tumors. $\left.{ }^{5}\right)$

In the present study, we investigated the effects of intravenously administered dCyd on tumor growth and survival time in SP2/0 tumor-bearing mice. Intravenous administration of dCyd clearly suppressed tumor growth and increased survival time in the tumor-bearing mice. The net amount of dCyd increased in the kidney, liver, and spleen of the tumor-bearing mice. Our results suggest that the increase in dCyd in the mice inoculated SP2/0 myeloma cells plays an important role for the growth suppression of the tumor.

\section{MATERIALS AND METHODS}

Chemicals Alkaline phosphatase-conjugated goat antirat immunoglobulin, dCyd, and 5-fluorouracil (5FU) were obtained from Sigma-Aldrich (St. Louis, MO, U.S.A.). We purchased RPMI 1640 medium from Nikken Biomedical Laboratory (Kyoto, Japan), fetal bovine serum (FBS) from Sanko Junyaku (Tokyo, Japan), penicillin and streptomycin from Meiji Seika Co. (Tokyo, Japan), phosphate-buffered saline (PBS) from Takara Shuzo Co., Ltd. (Shiga, Japan), and trypan blue, $\mathrm{HClO}_{4}$, and $\mathrm{KOH}$ from Wako Pure Chemical Industries, Ltd. (Osaka, Japan). We used Otsuka normal saline (Otsuka Co., Ltd., Tokyo, Japan) as sterile saline. Glycine was obtained from Nacalai Tesque (Kyoto, Japan).

Equipment Plastic tissue culture dishes, $100 \mathrm{~mm}$ in diameter, were purchased from IWAKI Co. (Tokyo, Japan). Filter units (Millex TM-GV 0.22- $\mu \mathrm{m}$, sterile and Millex TM-HV $0.45-\mu \mathrm{m}$, non-sterile) were purchased from Millipore Corp. (Bedford, MA, U.S.A.), and Supelclean LC-SAX SPE tubes were from Supelco (Bellefonte, PA, U.S.A.).

Cell Cultures of SP2/0-Ag14 Myeloma Cells SP2/0 cells were obtained from the Collection of Cancer Cell Lines (National Institute of Hygienic Science, Tokyo, Japan). The cells were maintained in RPMI medium containing $10 \%$ heat-inactivated FBS, penicillin $(100 \mathrm{U} / \mathrm{mL})$, and streptomycin $(100 \mu \mathrm{U} / \mathrm{mL})$ and cultured in a humidified atmosphere of $5 \%$ $\mathrm{CO}_{2}$ at $37^{\circ} \mathrm{C}$. To inoculate the mice, cells were removed from the dishes by pipetting and washed twice with PBS. The cell number was determined based on trypan blue exclusion.

Animals and Tumor Volume Measurement BALB/c mice (male, 5 weeks old, weighing $20-25 \mathrm{~g}$ ) were obtained from Japan SLC (Hamamatsu, Japan). The animals were continually cared for in a room maintained at $25 \pm 1{ }^{\circ} \mathrm{C}$ and $55 \pm 5 \%$ humidity, with free access to water and food. The protocols were performed according to the guidelines of the Japanese Society for Pharmacology and were approved by the Committee for Ethical Use of Experimental Animals at Setsunan University.

For tumor-bearing treatment groups, SP2/0 cell suspension $\left(1 \times 10^{6}\right.$ cells/mouse) was inoculated subcutaneously into a shaved area on the back of each mouse on day 0 . Seven days later, body weight and tumor size were measured. Tumor volume was calculated using the following formula: tumor volume $\left(\mathrm{mm}^{3}\right)=A \times A \times B / 2$, where $A$ is the smallest diameter $(\mathrm{mm})$ and $B$ is the largest diameter $(\mathrm{mm})$ of the solid tumor, measured with vernier calipers. When the tumor volume reached approximately $100-300 \mathrm{~mm}^{3}$ at day 8 , the mice were administered saline, dCyd, or $5 \mathrm{FU}$ at $0.1 \mathrm{~mL} / 10 \mathrm{~g}$ body weight by intravenous injection according to the schedules below. $\mathrm{dCyd}$ and 5FU were dissolved in sterile saline and filtered through $0.22-\mu \mathrm{m}$ filters before injection.

Treatment with dCyd and 5FU Tumor-bearing mice received an intravenous injection of $\mathrm{dCyd}(25 \mathrm{mg} / \mathrm{kg})$ on days $8,10,12$, and 14 (T-dCyd), or $5 \mathrm{FU}(65 \mathrm{mg} / \mathrm{kg})$ on days 8 and 14 (T-5FU). The control group of tumor-bearing mice received saline intravenously on days $8,10,12$, and 14 . The mice were sacrificed under diethyl ether anesthesia $6 \mathrm{~d}$ after the final administration (day 20), and tissues and tumors were excised and weighed. Non-tumor-bearing mice received saline (normal) or dCyd (N-dCyd) intravenously on the same schedule as the control and T-dCyd groups, respectively. 5FU was used as a positive control for decreased tumor volume because it reduced SP2/0 tumor volume after intraperitoneal injection in 


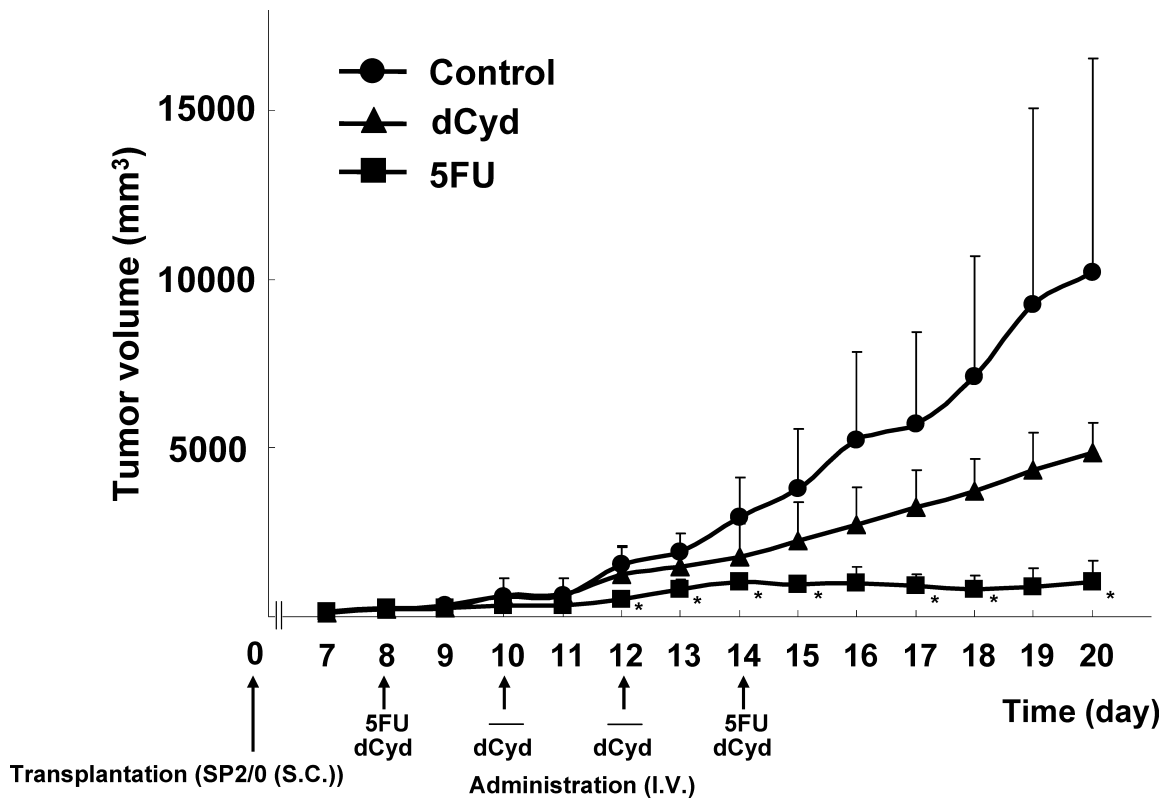

Fig. 1. Effects of dCyd and 5FU Administration on Tumor Volume

SP2/0 cell suspension $\left(1 \times 10^{6}\right.$ cells/mouse) was inoculated subcutaneously (s.c.) on the back of BALB/c mice on day 0 . Tumor-bearing mice received an intravenous injection of dCyd $(25 \mathrm{mg} / \mathrm{kg})$ on days $8,10,12$, and $14,5 \mathrm{FU}(65 \mathrm{mg} / \mathrm{kg})$ on days 8 and 14 , and the control group mice received saline at $0.1 \mathrm{~mL} / 10 \mathrm{~g}$ body weight intravenously on days $8,10,12$, and 14 . Tumor volume was calculated according to the Materials and Methods. ${ }^{*} p<0.05$, significantly different from control.

our previous study. ${ }^{5)}$

Measurement of dCyd in Tissues by Enzyme Immunoassay (EIA) dCyd was measured by EIA. Briefly, $\mathrm{HClO}_{4}$ ( $10 \mathrm{~mL}$ of a $10 \%$ solution) was added to $1 \mathrm{~g}$ (wet weight) of tissue, and the tissue was homogenized. The homogenate was centrifuged $(1500 \times \boldsymbol{g}, 15 \mathrm{~min})$, and the supernatant was applied to Supelclean ${ }^{\mathrm{TM}}$ LC-SAX SPE tubes that had been conditioned by washing with distilled water and $0.2 \mathrm{M}$ glycine buffer $(\mathrm{pH}$ 3.0). dCyd was eluted with glycine buffer, and the eluate containing dCyd was neutralized with $2 \mathrm{M} \mathrm{KOH}$. After centrifugation $(500 \times \boldsymbol{g}, 15 \mathrm{~min})$, the supernatant was lyophilized. The sample was solubilized in $0.1 \mathrm{~m}$ phosphate buffer, $\mathrm{pH} 7.2$, passed through a $0.45-\mu \mathrm{m}$ filter, and subjected to EIA, according to the method described by Darwish et al. ${ }^{\text {) }}$

Determination of Survival Time of Tumor-Bearing Mice dCyd at 2.5, 25, and $100 \mathrm{mg} / \mathrm{kg}$ was administered intravenously to tumor-bearing mice on day 8 . Control animals received saline. Each group consisted of four or five mice. Survival was evaluated.

Statistical Analysis Data were analyzed using Student's or Welch's $t$-test, the $F$-test, and the Mann-Whitney $U$ test, with Statcel2 software. Survival curves were calculated using the Kaplan-Meier method, and differences in survival were analyzed using the log-rank test.

\section{RESULTS}

Effects of dCyd and 5FU Administration on Tumor Volume and Weight Tumor volume was not significantly different among the groups until day 11 (Fig. 1). At day 12, the tumor volume was significantly smaller in the 5FU group $\left(500 \mathrm{~mm}^{3}\right)$ than in the control group $\left(1500 \mathrm{~mm}^{3}\right)$. At day 20, the tumor volume was significantly smaller in the 5FU group $\left(1000 \mathrm{~mm}^{3}\right)$ than in the control group $\left(10000 \mathrm{~mm}^{3}\right)$ and the dCyd group $\left(4800 \mathrm{~mm}^{3}\right)$, which had about half the volume of the control.

At day 20, the tumor weights were significantly less in the dCyd $(2.1 \mathrm{~g})$ and $5 \mathrm{FU}$ groups $(0.25 \mathrm{~g})$ than in the control $(3.8 \mathrm{~g})$. Among the three groups, the 5FU group had the lowest tumor weight (Fig. 2A), consistent with the tumor volume results.

Weight and dCyd Levels in Tumors and Tissues Figure 2 shows the weights and dCyd levels ( $\mu \mathrm{mol} / \mathrm{g}$ wet weight) of the tumors (A), kidneys (B), livers (C), and spleens (D) in non-tumor-bearing and tumor-bearing mice. The tissue weights of the kidney (Fig. 2B), heart, and lung (data not shown) did not differ between the non-tumor-bearing and tumor-bearing mice, or between the control and the T-dCyd or T-5FU group. However, the weights of the liver (Fig. 2C) and spleen (Fig. 2D) in the control group were 1.4- and 3.8-fold, respectively, those in the normal group. The liver weight was lower in the T-dCyd and T-5FU groups than in the control group. The spleen weight was nearly the same in the T-5FU and normal groups.

The dCyd levels in the kidney, liver, spleen, and tumor were between 1.5 and $6.2 \mu \mathrm{mol} / \mathrm{g}$. In the kidney, the $\mathrm{dCyd}$ level in the control was 2.5 -fold that in the normal group. The level returned to normal with 5FU administration. The dCyd level in the N-dCyd group was higher than that in the normal group, but the dCyd level in the T-dCyd group was not higher than that in the control group. The dCyd levels in the liver and spleen of tumor-bearing mice were not different from those in the normal group, and the levels in the dCyd-treated group did not differ from those in the normal or control group.

In the tumor-bearing mice, the net amounts of $\mathrm{dCyd}(\mu \mathrm{mol} /$ tissue) in the kidney, liver, and spleen increased 3.0-, 2.5-, and 5.3-fold, respectively, compared with the levels in the nontumor-bearing mice (Table 1). The net amount of dCyd in the tumors $(18 \mu \mathrm{mol} /$ tumor) was higher than that in the other tissues owing to the greater weight of the tumor compared with 

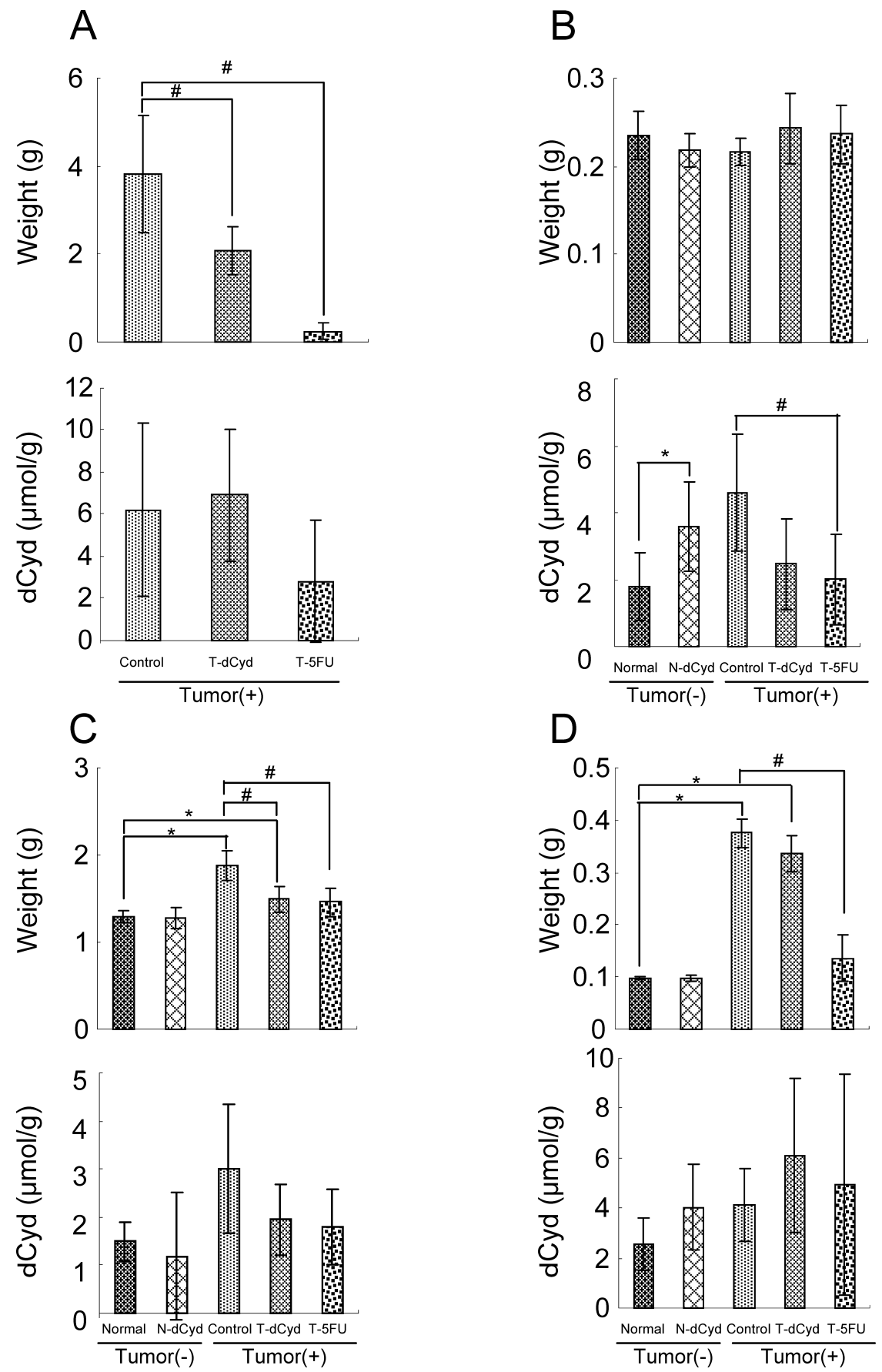

Fig. 2. Weights and dCyd Levels of Tumor (A), Kidney (B), Liver (C), and Spleen (D)

The administration schedule is as follows in Fig. 1. Tumor-bearing mice were injected dCyd ( $25 \mathrm{mg} / \mathrm{kg}$ ) (T-dCyd), $5 \mathrm{FU}(65 \mathrm{mg} / \mathrm{kg})$ (T-5FU), or saline (control). Nontumor-bearing mice were injected saline (normal) or dCyd (N-dCyd). The mice were sacrificed under diethyl ether anesthesia at $6 \mathrm{~d}$ after the final administration (day 20 ), and tissues and tumors were excised and weighed. dCyd level was measured according to the Materials and Methods. ${ }^{*} p<0.05$, significantly different from normal (nontumor-bearing controls). ${ }^{\#} p<0.05$, significantly different from control (tumor-bearing controls).

the other tissues. In the heart and lung, the dCyd level and net amount of dCyd did not differ between non-tumor-bearing and tumor-bearing mice (data not shown).

Survival Analysis of Tumor-Bearing Mice The effect of dCyd on survival time of tumor-bearing mice is shown in Fig. 3. A single injection of $25 \mathrm{mg} / \mathrm{kg}$ or $100 \mathrm{mg} / \mathrm{kg}$ of dCyd significantly increased survival time compared with that of the control group. The $50 \%$ survival times were as follows: control group, $38 \mathrm{~d} ; 2.5 \mathrm{mg} / \mathrm{kg}$ dCyd group, $44 \mathrm{~d} ; 25 \mathrm{mg} / \mathrm{kg}$ dCyd group, $43 \mathrm{~d}$; and $100 \mathrm{mg} / \mathrm{kg}$ dCyd group, $42 \mathrm{~d}$.

\section{DISCUSSION}

We examined the effect of intravenously injected dCyd on tumor growth. At day 20 (6d after the final injection), the tumor weight was less in the dCyd group than in the control group.

In our previous study, intraperitoneal administration of dCyd tended to decrease tumor growth in mice subcutaneously transplanted with SP2/0 mouse myeloma cells. ${ }^{5)}$ As dCyd was administered by intravenous injection in the current 
study, it was present in the blood at a higher concentration, and the suppressive effect of dCyd on tumor growth was clear and significant. In the previous study, dCyd was administered by daily intraperitoneal injections at a dose of $25 \mathrm{mg}$ / $\mathrm{kg}(0.1 \mathrm{mmol} / \mathrm{kg})$ on days $8-15$. In the present study, dCyd was administered by intravenous injection at a dose of $25 \mathrm{mg} /$ $\mathrm{kg}$ on days $8,10,12$ and 14 . Thus, the total intravenous dose $(100 \mathrm{mg} / \mathrm{kg})$ was half of the total intraperitoneal dose $(200 \mathrm{mg} /$ $\mathrm{kg}$ ), indicating that dCyd is effective at a lower dose when administered intravenously rather than intraperitoneally.

Notably, a single intravenous administration of dCyd increased survival time in the tumor-bearing mice. This effect appeared to be stronger with the $25 \mathrm{mg} / \mathrm{kg}$ dose of $\mathrm{dCyd}$, although survival time did not depend on the dCyd dose. These results suggest that the suppressive effect of dCyd on tumor growth continues after the final administration.

Moyer et $a l^{7)}$ studied the metabolism of radiolabeled pyrimidine nucleosides administered intravenously to mice. They observed that the half-life of dCyd was less than $9 \mathrm{~min}$ and that deoxyuridine (dUrd), formed from the deamination of dCyd, was present even after $1 \mathrm{~min}$ and equaled the plasma concentration of dCyd by $10 \mathrm{~min}$. Additionally, they measured the radioactivity of the nucleoside in the acid-soluble fraction and the DNA fraction of tissues. After $30 \mathrm{~min}$, labeled

Table 1. Net Amounts of dCyd in Tissues and Tumors in BALB/c Mice with and without Inoculation of SP2/0 Mouse Myeloma Cells

\begin{tabular}{lcc}
\hline \hline Tissue & Tumor & $\begin{array}{c}\text { Net amount of dCyd } \\
(\mu \mathrm{mol} / \text { tissue })\end{array}$ \\
\hline Kidney & - & $0.41 \pm 0.18$ \\
& + & $1.01 \pm 0.40^{*}$ \\
Liver & - & $1.91 \pm 0.40$ \\
& + & $5.79 \pm 2.93^{*}$ \\
Spleen & - & $0.27 \pm 0.10$ \\
& + & $1.43 \pm 0.54^{*}$ \\
Solid tumor & & $17.76 \pm 5.02$
\end{tabular}

- : Non-tumor-bearing mice. +: Tumor-bearing mice. The net amount of dCyd was calculated by the level of dCyd times the tissue or tumor weight. Each value represents the mean \pm S.D. $* p<0.05$, significantly different from non-tumor-bearing mice.
dCyd was detected extensively in the DNA of the intestine and spleen. The tissues investigated contained unmetabolized $\mathrm{dCyd}$ at less than $3 \%$ of the total acid-soluble radiolabel. This report suggested that dCyd was removed from the plasma almost immediately after administration and was distributed in tissues in the form of dUrd and/or DNA. In the present study, the administered dCyd might not have been detectable in plasma because the dCyd level was measured $6 \mathrm{~d}$ after the final injection.

Schwarzenberg et $a l .{ }^{8)}$ investigated the distributions of positron emission tomography (PET) probes (analogs of the anti-cancer agent gemcitabine and $\mathrm{dCyd}$ ) in humans $17-79 \mathrm{~min}$ after administration. ${ }^{18} \mathrm{~F}-1-\left(2^{\prime}\right.$-deoxy-2'-fluoroarabinofuranosyl)cytosine $\left({ }^{18} \mathrm{~F}-\mathrm{FAC}\right)$ was trapped at high levels in the spleen and kidney following phosphorylation by deoxycytidine kinase $(\mathrm{dCK})$ and was catabolized by cytidine deaminase (CDA). 1-(2'-Deoxy-2'-1 ${ }^{\prime}$ F-fluoro-arabinofuranosyl)cytosine $\left(\mathrm{L}-{ }^{18} \mathrm{~F}-\mathrm{FAC}\right)$ was trapped at high levels in the liver following phosphorylation, but it was not catabolized by CDA. Osogoe et al. reported that after intraperitoneal administration of tritiated dCyd, radioactivity was strongly detected in the germinal center cells of the spleen and in Peyer's patches of the intestine in both mice ${ }^{9)}$ and rats. ${ }^{10)}$ These findings suggest that kidney, liver, and spleen may be the major sites of $\mathrm{dCyd}$ accumulation initially after administration and that dCyd may be catabolized in each tissue following administration. In the present study, the administration of dCyd did not affect the $\mathrm{dCyd}$ levels in the heart, lung, liver, or spleen of tumor-bearing or non-tumor-bearing mice at day 20. However, based on the findings of Schwarzenberg et al. ${ }^{8)}$ and Osogoe et al., ${ }^{9,10)}$ it was expected that administered dCyd would be distributed to the spleen during the early stages after administration.

We believe the antitumor effect of dCyd occurs via direct and indirect mechanisms, and we are conducting in vitro experiments to further clarify the direct suppressive effects of dCyd on tumor cell growth. dCyd may indirectly affect nucleoside transport and/or the enzymatic activities of $\mathrm{dCK}$ and CDA. This could explain the continued suppressive effect of dCyd on tumor growth after the final administration.

Muramatsu et al. ${ }^{11)}$ reported that the aberrant activation-

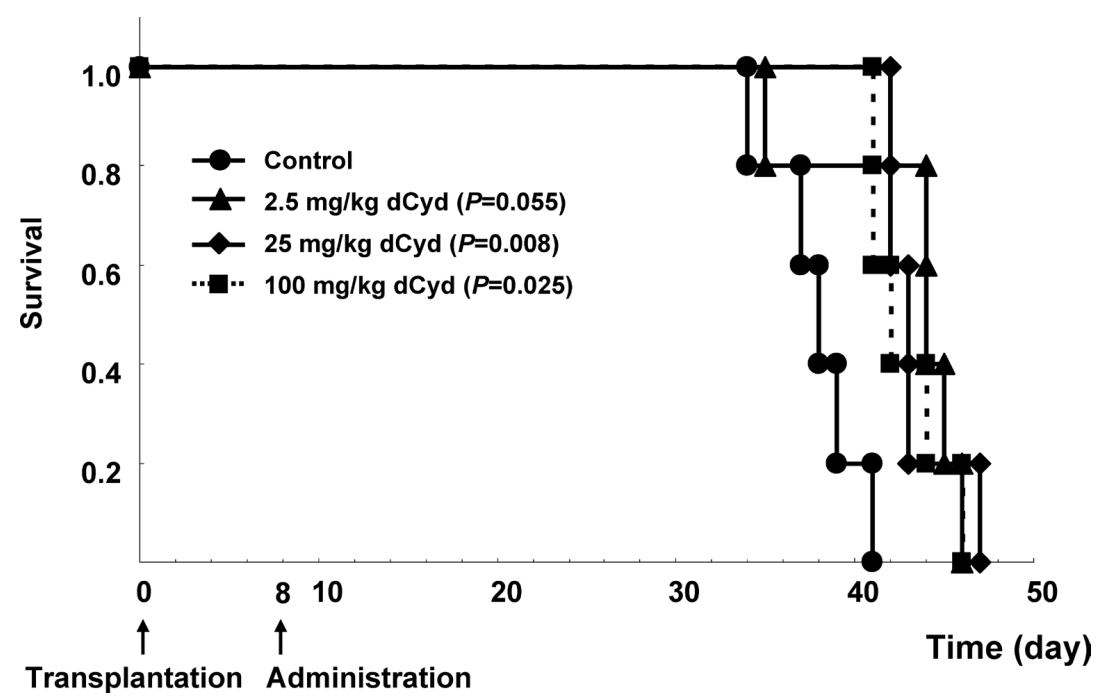

Fig. 3. Effects of dCyd on Cancer-Induced Death (Kaplan-Meier Plot)

dCyd was administered intravenously at $2.5,25$, and $100 \mathrm{mg} / \mathrm{kg}$ to tumor-bearing mice on day 8 . Control animals received saline. $p$-Values are for the log-rank test. 
induced cytidine deaminase (AID) gene was specifically expressed in germinal center B cells of the spleen, a secondary lymphoid organ. AID is a member of the CDA family, for which cytidine and dCyd are substrates, and is essential in somatic hypermutation and class switching recombination of immunoglobulin genes. ${ }^{12)}$ We suspect that immunity may be influenced by the increased concentration of dCyd in the tumor-bearing spleen. The net amount of dCyd in the spleen was increased in the tumor-bearing mice compared with the non-tumor-bearing mice, consistent with previous results. ${ }^{5)}$ Schwarzenberg et al. ${ }^{8)}$ reported that ${ }^{18}$ F-FAC catabolized by CDA was trapped at high levels in the kidney and spleen. Thus, administered dCyd may accumulate in the spleen soon after administration and/or the dCyd increase in tumor-bearing mice may influence the immunity effect of CDA.

Our results suggest that the increase in dCyd in the mice inoculated SP2/0 myeloma cells plays an important role for the growth suppression of the tumor. Our laboratory is undertaking further studies to clarify the role of dCyd in the tumorbearing body.

Acknowledgements We thank Ms. C. Kikkawa, Ms. H. Morita, and Ms. Y. Yagawa for their technical assistance.

\section{REFERENCES}

1) Silagi S. Metabolism of 1- $\beta$-D-arabinofuranosylcytosine in $\mathrm{L}$ cells. Cancer Res., 25, 1446-1453 (1965).

2) Heinemann V, Hertel LW, Grindey GB, Plunkett W. Comparison of the cellular pharmacokinetics and toxicity of $2^{\prime}, 2^{\prime}$-difluorodeoxycytidine and 1- $\beta$-D-arabinofuranosylcytosine. Cancer Res., 48, 4024-4031 (1988).

3) Hertel LW, Boder GB, Kroin JS, Rinzel SM, Poore GA, Todd GC,
Grindey GB. Evaluation of the antitumor activity of gemcitabine (2',2'-difluoro-2'-deoxycytidine). Cancer Res., 50, 4417-4422 (1990).

4) Momparler RL, Goodman J. In vitro cytotoxic and biochemical effects of 5-aza-2'-deoxycytidine. Cancer Res., 37, 1636-1639 (1977).

5) Iwazaki A, Imai K, Nakanishi K, Yoshioka M. Changes in 2'-deoxycytidine levels in various tissues of tumor-bearing mice. Oncol. Lett., 1, 999-1004 (2010).

6) Darwish I, Akizawa T, Hirose K, Omura K, EL-Rabbat N, Yoshioka M. Preparation of a specific monoclonal antibody against 2'-deoxycytidine. Anal. Chim. Acta, 365, 121-128 (1998).

7) Moyer JD, Malinowski N, Ayers O. Salvage of circulating pyrimidine nucleosides by tissues of the mouse. J. Biol. Chem., 260, 2812-2818 (1985).

8) Schwarzenberg J, Radu CG, Benz M, Fueger B, Tran AQ, Phelps ME, Witte ON, Satyamurthy N, Czernin J, Schiepers C. Human biodistribution and radiation dosimetry of novel PET probes targeting the deoxyribonucleoside salvage pathway. Eur. J. Nucl. Med. Mol. Imaging, 38, 711-721 (2011).

9) Osogoe B, Ueki A. A radioautographic study of the utilization of deoxycytidine for the formation of deoxyribonucleic acid-thymine in lymphocytes. J. Cell Biol., 46, 403-405 (1970).

10) Osogoe B, Tyler RW, Everett NB. The patterns of labeling of germinal-center cells with tritiated deoxycytidine. J. Cell Biol., 57, 215-220 (1973).

11) Muramatsu M, Sankaranand VS, Anant S, Sugai M, Kinoshita K, Davidson NO, Honjo T. Specific expression of activation-induced cytidine deaminase (AID), a novel member of the RNA-editing deaminase family in germinal center B cells. J. Biol. Chem., 274, 18470-18476 (1999).

12) Muramatsu M, Kinoshita K, Fagarasan S, Yamada S, Shinkai Y, Honjo T. Class switch recombination and hypermutation require activation-induced cytidine deaminase (AID), a potential RNA editing enzyme. Cell, 102, 553-563 (2000). 